Dig Surg 1996;13:442

\title{
Subject Index Vol. 13, No. 4-5,1996
}

\section{Acidosis 255}

Acute acalculous cholecystitis 328

appendicitis 304

calculous cholecystitis 328

cholecystitis 328, 332

necrotizing pancreatitis 357

pancreatitis 269, 362, 365, 370, 374

respiratory distress syndrome 273

- - -, clinical picture 273 Adrenergic agents 245 Algorithm 374

Antibiotic prophylaxis 370

$$
\text { - } \quad \text { treatment } 365
$$

Antibiotics 287, 317, 346, 357, 374 Antimicrobial agents 421

prophylaxis 301

therapy 421 APACHE II 381 Appendectomy 301,309 Appendicitis 287,301,309

Bacteremia 317

Bacteria 357

Bacterial pathogenesis 390

resistance 421

translocation 250, 261, 269, 362 Bactibilia 317

Bile 317

Biliary obstruction 321

- $\quad$ tract surgery 287

C-reactive protein 412 Care-related stress factors 439 Catheter-related infection 412 Cholangitis

314,317,321 Cholecystectomy 321 Cholecystitis 314,317 Cholecystostomy 328

Choledocholithiasis 321 Classification 381 Clean surgery 287 Colon surgery 287 Colonic

diverticulitis 349 Computed tomography 338 Conservative approaches 309 Contrast enema 338

Cost analysis 301 Critical illness 277 Cytokines 245

Diagnosis 309,314,346 Diffuse peritonitis 396 Diverticulitis 338, 346, 353 Diverticulosis 338

Endogenous infections 294 Endoscopic retrograde cholangiopancrea-tography 321

Endotoxemia 261 Endotoxin 261,384 Enteral feeding 410

- $\quad$ nutrition 245

Enterocytes 250

Esophagus 273

Etappenlavage 396

Etiology 381

Fever 417

-, postoperative 412

Flowmotion 250

Gastroduodenal surgery 287 
Glutamine 277

Gut permeability 245

Hypoxia 245, 255

IgG 430 Imaging 338

Immunoglobulin therapy 430 Infected bile 362

- $\quad$ pancreatic necrosis 370

Infection 357

Intensive care 294, 410, 421, 439

- $\quad$ - unit 417,435

Interleukin-6 412

Intramucosal pH 255

Intravenous immune globulin 435

Klebsiella spp. 435

Laparoscopic cholecystectomy 328, 332

Laparoscopy 321

Laparotomy 309

Lavage 374,400

Length of hospitalization 405

Mannheimer Peritonitis Index 381 Mediators 245 Medical ethics 439

- $\quad$ treatment failure 349

Meta-analyses 309

Microbiology 282

Morbidity 332

Mortality 332, 374

Mucosa 250

Multiple-organ dysfunction syndrome 261

- $\quad$ failure 250, 277, 384, 425

Multiple trauma 405

Necrosectomy 374

Necrosis 357

Necrotizing pancreatitis 374

Nitric oxide 425

Nosocomial infection(s) 412, 417, 435

- $\quad$ pneumonia 405

Open cholecystectomy 328, 332 Operation, delayed 328 -, early 328

Pancreatic tissue concentrations 365 Pelvic abscess computed tomography 349 Peritonitis 282, 381, 384, 390, 400 Pharmacoeconomics 405 Pharmacokinetics 365 Pneumonia 412

Premedication 287 Primary anastomosis 353 Prophylaxis 410 Pseudomonas 317

- $\quad$ aeruginosa 435

Radiography 338 Ranitidine prophylaxis 405 Resistant pathogens 282 Reticuloendothelial system dysfunction 362 Risk factors 405

Scheduled reoperations 396 Score 309

Selective decontamination 294 Sepsis 245, 250, 255, 261, 269, 314, 384, 425,430

score 430

syndrome 425 Septic shock 255, 425, 430 Shock 250

Single-stage procedure 353 Splanchnic blood flow 245 
ischaemia 255 Standard therapy 328 Stress ulcer 410 Surgery 261,346,374 Surgical infections 430

strategy 353

wound infection 287

Systemic inflammatory response syndrome 250,417

Therapeutic concepts 396 Tonometry 255 Translocation 245,261 Trauma 250 Treatment 346, 390, 400

Ultrasonography 304 Ultrasound 309, 338 Upper gastrointestinal bleeding 405

Vasomotion 250 Ventilator-associated pneumonia 417

442 